\title{
FAMILY OF TWO-DIMENSIONAL BORN-INFELD EQUATIONS AND A SYSTEM OF CONSERVATION LAWS
}

\author{
(Presented by H. Keres)
}

Lower-order conserved quantities, the «currents», for two-dimensional Lorentz-invariant Born-Infeld equation are considered. The currents are divided into pairs, which contain a class (basic currents) leading to the family of equations. The basic currents determine the transformations between the solutions of the Born-Infeld equation family. The equations belonging to the family are fully hodograph-invariant, partly hodographinvariant, and effectively linear, i. e. non-linear equations with linear image of hodograph transformation.

Two-dimensional non-linear equations with soliton solutions possess an infinite series of s. c. topological conservation laws. This system can be understood in terms of completely integrable Hamilton systems ['].

The conservation laws represent a kind of generalization on energymomentum conservation and are free-field properties that still hold in non-linear region. It is easy to see that for linear equation $\square \varphi=0$. $\left(\square=\partial_{x}^{2}-\partial_{t}^{2}\right)$ with the law of energy conservation (the conservation of «lowest current») $F_{t}=G_{x} ; F^{0}=\varphi_{x}^{2}+\varphi_{t}^{2}, G^{0}=2 \varphi_{x} \varphi_{t}$ there is an infinite system on «higher currents» that are also conserved: $F^{k}=\varphi_{(k+1) x}^{2}+\varphi_{h x, t}^{2}$, $G^{k}=2 \varphi_{(k+1) x} \varphi_{k x, t} \quad$ (where $\varphi_{k x}$ is the $k$-th derivative).

There are well-known systems of higher currents for Korteweg - de Vries, Sine-Gordon and for all «classical» soliton equations.

On the other hand, for the same linear equation $\square \varphi=0$ the lowest currents may be taken in an infinite number of ways. In general the lowest currents for $\square \varphi=0$ have the form

$$
\begin{aligned}
& F=f\left(\varphi_{t}+\varphi_{x}\right)+g\left(\varphi_{t}-\varphi_{x}\right), \\
& G=f\left(\varphi_{t}+\varphi_{x}\right)-g\left(\varphi_{t}-\varphi_{x}\right),
\end{aligned}
$$

where $f$ and $g$ are arbitrary functions. We get the energy conservation if we take $f(x)=g(x)=x^{2}$. As far as $F_{t}=G_{x}$, we may take $\psi_{x}=F$ and $\psi t=G$ and it is easy to see that for $\psi \square \psi=0$ too. Therefore the equation (A) can be considered as a transformation from the solution of $\square \varphi=0$ to another.

In non-linear relativistic cases we can see that the arbitrariness of choosing lower currents reduces drastically. For Sine-Gordon equation $\square \varphi=\sin \varphi$, we see that $F^{0}$ and $G^{0}$ may be chosen «only» in the forms 


$$
\begin{aligned}
& \psi_{x}+\psi_{t}=A\left(\varphi_{t}+\varphi_{x}\right)^{2}+2 B \cos \varphi, \\
& \psi_{x}-\psi_{t}=B\left(\varphi_{t}-\varphi_{x}\right)^{2}+2 A \cos \varphi,
\end{aligned}
$$

where the function $\psi$ generally does not satisfy the Sine-Gordon equation, it even cannot be determined as a solution of a certain differential equation. The transformations analogous to $(\mathrm{A})$, which transform one of the solutions of an equation to another (Bäcklund transformation for Sine-Gordon equation), are, as it is well known, $\psi$-dependent i.e. $\psi x$ cannot be put into a form $F\left(\varphi, \varphi_{x}, \varphi_{t}\right)$ but into $F\left(\varphi, \varphi_{x}, \varphi_{t}, \psi\right)$. Therefore, for «classical» soliton equations the system of lowest currents is of little interest.

For the case of the two-dimensional «relativistic» Born-Infeld equation on the contrary, the «spectrum» of lowest currents is very rich and leads to several interesting conclusions. Henceforth we shall call the «lowest currents» simply «currents».

The main subject and the starting point of this investigation is the well-known two-dimensional Born - Infeld scalar equation [ [ $\left.{ }^{2}\right]$

$$
\left(1-\varphi_{t}^{2}\right) \varphi_{x x}+2 \varphi_{x} \varphi_{t} \varphi_{x t}-\left(1+\varphi_{x}^{2}\right) \varphi_{t t}=0 .
$$

In Euclide metric this hyperbolic equation transforms into elliptic (minimal surface) equation $(x \rightarrow x, t \rightarrow i y)$

$$
\left(1+\Phi_{y}^{2}\right) \Phi_{x x}-2 \Phi_{x} \Phi_{y} \Phi_{x y}+\left(1+\Phi_{x}^{2}\right) \Phi_{y y}=0 .
$$

Equation (2) has a remarkable property of being «hodograph invariant», i. e. it does not change its form by the hodograph transformations $\Phi(x, y) \rightarrow x(y, \Phi)$ and $\Phi(x, y) \rightarrow y(\Phi, x)$. Equation (1) has the same property under the hodograph transformation $\varphi(x, t) \rightarrow x(\varphi, t)$, but for $\varphi(x, t) \rightarrow t(\varphi, x)$ there is a change of signature

$$
\left(1-t_{\varphi}^{2}\right) t_{x x}+2 t_{\varphi} t_{x} t_{\varphi x}+\left(1-t_{x}^{2}\right) t_{\varphi \varphi}=0 .
$$

We can say that equation (2) is hodograph invariant with respect to both arguments. Up to signature, the same is valid for equation (1). (Equation (1) can be called «hodograph invariant up to signature».)

Equation (1) has three pairs of conserved quantities $(F, G)$ («currents» $F_{t}=G_{x}$ ), which further will be called the «basic». To see this, it is convenient to write (1) as a system of exterior forms $\alpha_{i}$.

Let $z=\varphi_{x}, p=\varphi_{t}$ and let

$$
\begin{aligned}
& \alpha_{1}=z d x \wedge d t-d \varphi \wedge d t, \\
& \alpha_{2}=p d x \wedge d t-d x \wedge d \varphi, \\
& \alpha_{3}=\left(1-p^{2}\right) d z \wedge d t+z p d x \wedge d z+z p d p \wedge d t-\left(1+z^{2}\right) d x \wedge d p, \\
& \alpha_{4}=d p \wedge d t+d z \wedge d x,
\end{aligned}
$$

where $d x, d t, d \varphi, d z, d p$ are 5 independent 1 -forms. The manifold of equation (1) solutions is obtained as a submanifold with independent forms $d x$ and $d t$, which annihilates the system of $\alpha_{i}$-s.

Now consider the 1 -forms $w_{k}=F^{k} d x+G^{k} d t \quad\left(F^{k}=F^{k}(\varphi, p, z) ; \quad G^{k}=\right.$ $\left.=G^{k}(\varphi, p, z)\right)$, for which:

$$
d w_{k}=f_{i}^{k} \alpha_{i},
$$


where $f_{i}^{k}$-s are arbitrary functions of $\varphi, p$ and $z$. In the submanifold of solutions $\left(\alpha_{i}=0\right)$

$$
d w_{k}=\left(F_{t}^{k}-G_{x}^{k}\right) d t \wedge d x=0 .
$$

Putting $w_{k}=F^{k} d x+G^{k} d t$ and $\alpha_{i}$-s in (3) and eliminating $f_{i}^{k}$-s, we obtain for $F^{k}$ and $G^{k}$

$$
\begin{aligned}
& \left(1+z^{2}\right) F_{z z}^{k}+2 p z F_{p z}^{k}+\left(p^{2}-1\right) F_{p p}^{k}+4 p\left(1+z^{2}\right)^{-1} F_{p}^{k}=0, \\
& G_{z}^{k}=\left(1-p^{2}\right)\left(1+z^{2}\right)^{-1} F_{p}^{k}, \quad G_{p}^{k}=F_{z}^{k}+2 z p\left(1+z^{2}\right)^{-1} F_{p}^{k} .
\end{aligned}
$$

The basic currents satisfying (4) are

$$
\begin{aligned}
& F^{1}=z, \quad G^{1}=p ; \\
& F^{2}=\left(1+z^{2}\right) /\left(1-p^{2}\right), \quad G^{2}=2 z p /\left(1-p^{2}\right) ; \\
& F^{3}=\left(1+z^{2}\right) / \sqrt{1+z^{2}-p^{2}}, \quad G^{3}=z p / \sqrt{1+z^{2}-p^{2}} ; \\
& \tilde{F}^{1}=p / \sqrt{1+z^{2}-p^{2}}, \quad \widetilde{G}^{1}=z / \sqrt{1+z^{2}-p^{2}}, \\
& F^{2}=2 z p /\left(1+z^{2}\right), \quad \bar{G}^{2}=\left(p^{2}-1\right) /\left(1+z^{2}\right), \\
& \tilde{F}^{3}=z p / \sqrt{1+z^{2}-p^{2}}, \quad \widetilde{G}^{3}=\left(p^{2}-1\right) / \sqrt{1+z^{2}-p^{2}} .
\end{aligned}
$$

Another, nonbasic pair is

$$
\begin{aligned}
& F^{4}=p\left(1+z^{2}\right) /\left(1-p^{2}\right), \quad G^{4}=z\left(1+p^{2}\right) /\left(1-p^{2}\right) ; \\
& \widetilde{F}^{4}=p\left(z^{2}-1\right) /\left(1+z^{2}\right), \tilde{G}^{4}=z\left(p^{2}-1\right) /\left(1+z^{2}\right) .
\end{aligned}
$$

Before going into details, let us note the following: the quantity $\dot{F}^{3}$ is the Hamiltonian of equation (1) and represents the conservation law for energy $\int F^{3} d x, \int F^{1} d x=$ const expresses the conservation of canonical momentum. The pair of currents $\left(F^{2}, G^{2}\right),\left(F^{2}, G^{2}\right)$ determines isothermic coordinates $\left[{ }^{3}\right]$ on a minimal surface

$$
\begin{gathered}
g_{\alpha \alpha}+g_{\beta \beta}=0, \\
g_{\alpha \beta}=0,
\end{gathered}
$$

where $g_{\sigma \rho}=\varphi_{\sigma} \varphi_{\rho}+x_{\sigma} x_{\rho}-t_{\sigma} t_{\rho}$ is the metric tensor of surface

$$
d s^{2}=g_{\alpha \alpha} d \alpha^{2}+g_{\alpha \beta} d \alpha d \beta+g_{\beta \beta} d \beta^{2},
$$

where $(\alpha, \beta)$ are the parameters of surface $\left(u^{0}=\alpha, u^{1}=\beta\right)$. Conditions (7) are equivalent to

$$
\begin{gathered}
\left(1+\varphi_{x}^{2}\right) x_{\alpha} x_{\beta}+\varphi_{x} \varphi_{t}\left(x_{\alpha} t_{\beta}+x_{\beta} t_{\alpha}\right)-\left(1-\varphi_{t}^{2}\right) t_{\alpha} t_{\beta}=0, \\
\left(1+\varphi_{x}^{2}\right)\left(x_{\alpha}^{2}+x_{\beta}^{2}\right)+2 \varphi_{x} \varphi_{t}\left(x_{\alpha} t_{\alpha}+x_{\beta} t_{\beta}\right)-\left(1-\varphi_{t}^{2}\right)\left(t_{\alpha}^{2}+t_{\beta}^{2}\right)=0 \\
\left(x_{\alpha} \equiv \frac{\partial x}{\partial u^{0}}, \ldots\right),
\end{gathered}
$$

from which we get

$$
\begin{aligned}
& F^{2}=\left(1+z^{2}\right) /\left(1-p^{2}\right)=-\left(t_{\beta}^{2}-t_{\alpha}^{2}\right) /\left(x_{\beta}^{2}-x_{\alpha}^{2}\right) \equiv F(\alpha, \beta), \\
& G^{2}=2 z p /\left(1-p^{2}\right)=2\left(x_{\alpha} t_{\alpha}-x_{\beta} t_{\beta}\right) /\left(x_{\alpha}^{2}-x_{\beta}^{2}\right) \equiv G(\alpha, \beta) .
\end{aligned}
$$

As $G_{x}^{2}=F_{t}^{2}$, we may put $G^{2}=\psi_{t}$ and $F^{2}=\psi_{x}$. Then 


$$
\begin{gathered}
\psi_{\alpha}=\psi_{t} t_{\alpha}+\psi_{x} x_{\alpha}=\left[2\left(x_{\alpha} t_{\alpha}-x_{\beta} t_{\beta}\right) t_{\alpha}+\left(t_{\beta}^{2}-t_{\alpha}^{2}\right) x_{\alpha}\right] /\left(x_{\alpha}^{2}-x_{\beta}^{2}\right), \\
\psi_{\beta}=\psi_{t} t_{\beta}+\psi_{x} x_{\beta}=\left[2\left(x_{\alpha} t_{\alpha}-x_{\beta} t_{\beta}\right) t_{\beta}+\left(t_{\beta}^{2}-t_{\alpha}^{2}\right) x_{\beta}\right] /\left(x_{\alpha}^{2}-x_{\beta}^{2}\right)
\end{gathered}
$$

and on the surface $\psi$ satisfies the equation

$$
\psi_{\alpha \alpha}-\psi_{\beta \beta}=0 \text {, }
$$

i. e. the same equation as in the case of surface coordinates $x^{\mu}=\varphi, x, t$ in isothermic parametrization.

Now investigate our basic pairs of currents in some detail.

Take pairs (5a)

$$
\begin{array}{ll}
F^{1}=\varphi_{x} \equiv z, & F^{1}=p / \sqrt{1+z^{2}-p^{2}}, \\
G^{1}=\varphi_{t} \equiv p, & \bar{G}^{1}=z / \sqrt{1+z^{2}-p^{2}} .
\end{array}
$$

As $F_{t}=G_{x}$, we may put $F=u_{x}$ and $G=u_{t}$. The integrability condition $u_{x t}=u_{t x}$ takes the form

$$
\left(p / \sqrt{1+z^{2}-p^{2}}\right)_{t}=\left(z / \sqrt{1+z^{2}-p^{2}}\right)_{x}
$$

and gives equation (1): The pairs $\varphi_{\lambda} \equiv\left(\varphi_{x}, \varphi_{t}\right), u_{\lambda} \equiv\left(u_{x}, u_{t}\right)$ transform into one another:

$$
\begin{gathered}
\varphi_{\lambda}=R\left(u_{\lambda}\right), \\
1+u_{x}^{2}-u_{t}^{2}=1 /\left(1+\varphi_{x}^{2}-\varphi_{t}^{2}\right), \\
\varphi_{t}=u_{x} / \sqrt{1+u_{x}^{2}-u_{t}^{2}}, \quad \varphi_{x}=u_{t} / \sqrt{1+u_{x}^{2}-u_{t}^{2}}, \quad R^{2}=1 .
\end{gathered}
$$

The integrability condition for $\varphi\left(\varphi_{x t}=\varphi_{t x}\right)$ gives the Born - Infeld equation for $u$ and the transformation $\varphi_{\lambda}=R\left(u_{\lambda}\right)$, therefore, changes one minimal surface into another. For pairs $(5 \mathrm{~b})$ it can be taken similarly

$$
\begin{array}{ll}
\psi_{x}=\left(1+z^{2}\right) /\left(1-p^{2}\right), & \psi_{t}=2 z p /\left(1-p^{2}\right), \\
\chi_{x}=2 z p /\left(1+z^{2}\right), & \chi_{t}=\left(p^{2}-1\right) /\left(1+z^{2}\right) .
\end{array}
$$

There exists a simple interrelation between the pairs:

$$
\chi_{x}=\psi_{t} / \psi_{x}, \quad \chi_{t}=-1 / \psi_{x} \quad \text { or } \quad \psi_{x}=-1 / \chi_{t}, \quad \psi_{t}=-\chi_{x} / \chi_{t} .
$$

It means that $\psi$ and $\chi$ satisfy the equations

$$
\begin{aligned}
\left(\psi_{t} / \psi_{x}\right)_{t}= & \left(-1 / \psi_{x}\right)_{x} \text { and }\left(1 / \chi_{t}\right)_{t}=\left(\chi_{x} / \chi_{t}\right)_{x}, \\
& \psi_{x x}+\psi_{t} \psi_{x t}-\psi_{x} \psi_{t t}=0 \\
& \chi_{t t}-\chi_{x} \chi_{x t}+\chi_{t} \chi_{x x}=0 .
\end{aligned}
$$

Equations (13a) and (13b) are semi-invariant under the hodograph transformation, i. e. (13a) is invariant under the transformation $\psi(x, t) \rightarrow$ $\rightarrow x(\psi, t)$ and (13b) under the transformation $\chi(x, t) \rightarrow t(x, \chi)$. Consider now the pairs $(5 \mathrm{c})$, which can be taken in the form

$$
\begin{array}{ll}
\mu_{x}=\left(1+z^{2}\right) / \sqrt{1+z^{2}-p^{2}}, & \mu_{t}=z p / \sqrt{1+z^{2}-p^{2}}, \\
v_{x}=z p / \sqrt{1+z^{2}-p^{2}}, & v_{t}=\left(p^{2}-1\right) / \sqrt{1+z^{2}-p^{2}} .
\end{array}
$$

We have relations between $v_{x}, v_{t}$ and $\mu_{x}, \mu_{t}$ 


$$
v_{x}=\mu_{t}, \quad v_{t}=\left(\mu_{t}^{2}-1\right) / \mu_{x} ; \quad \mu_{t}=v_{x}, \quad \mu_{x}=\left(v_{x}^{2}-1\right) / v_{t},
$$

from which one can get for $\mu$ and $v$ the equations

or

$$
\mu_{t t}=\left[\left(\mu_{t}^{2}-1\right) / \mu_{x}\right]_{x} \text { and } v_{x x}=\left[\left(v_{x}^{2}-1\right) / v_{t}\right]_{t}
$$

and

$$
\left(1-\mu_{t}^{2}\right) \mu_{x x}+2 \mu_{x} \mu_{t} \mu_{x t}+\left(0-\mu_{x}^{2}\right) \mu_{t t}=0
$$

$$
\left(0-v_{t}^{2}\right) v_{x x}+2 v_{x} v_{t} v_{x t}+\left(1-v_{x}^{2}\right) v_{t t}=0
$$

We call the set of differential equations (1), (13a), (13b), (15a), (15b) the family of Born-Infeld equations.

Equations (15a) and (15b) are effectively linear. The hodograph transformations $\mu(x, t) \rightarrow x(\mu, t)$ and $v(x, t) \rightarrow t(x, v)$ give

$$
\begin{aligned}
& x_{\mu \mu}-x_{t t}=0, \\
& t_{v v}-t_{x x}=0 .
\end{aligned}
$$

Transformation (9) changes neither equation (1) nor the basic currents (5a) and $(5 b)$ :

$$
\begin{aligned}
& F^{2}\left(u_{x}, u_{t}\right)=\left(1+u_{x}^{2}\right) /\left(1-u_{t}^{2}\right)=\left(1+z^{2}\right) /\left(1-p^{2}\right)=F^{2}(z, p), \\
& F^{2}\left(u_{x}, u_{t}\right)=2 u_{x} u_{t} /\left(1+u_{x}^{2}\right)=2 z p /\left(1+z^{2}\right)=\widetilde{F}^{2}(z, p), \\
& F^{3}\left(u_{x}, u_{t}\right)=u_{x} u_{t} / \sqrt{1+u_{x}^{2}-u_{t}^{2}}=z p / \sqrt{1+z^{2}-p^{2}}=F^{3}(z, p), \\
& F^{3}\left(u_{x}, u_{t}\right)=\left(1+u_{x}^{2}\right) / \sqrt{1+u_{x}^{2}-u_{t}^{2}}=\left(1+z^{2}\right) / \sqrt{1+z^{2}-p^{2}}=F^{3}(z, p) .
\end{aligned}
$$

The same holds for $G$-s.

From currents (6), however, by means of $R$ we get new conserved quantities $\left(F^{5}, G^{5}\right)$ and $\left(\widetilde{F}^{5}, \widetilde{G}^{5}\right)$

$$
\begin{aligned}
& \begin{aligned}
F^{4}\left(u_{x}, u_{t}\right) & =u_{t}\left(1+u_{x}^{2}\right) /\left(1-u_{t}^{2}\right)=z\left(1+z^{2}\right) /\left(1-p^{2}\right) \sqrt{1+z^{2}-p^{2}} \equiv F^{5}(z, p), \\
F^{4}\left(u_{x}, u_{t}\right)=u_{t}\left(u_{x}^{2}-1\right) /\left(1+u_{x}^{2}\right) & =z\left(2 p^{2}-1-z^{2}\right) /\left(1+z^{2}\right) \sqrt{1+z^{2}-p^{2}} \equiv \\
& \equiv F^{5}(z, p) .
\end{aligned} \\
& G^{5}(z, p)=p\left(1+2 z^{2}-p^{2}\right) /\left(1-p^{2}\right) \sqrt{1+z^{2}-p^{2}}, \\
& G^{5}(z, p)=-p\left(1-p^{2}\right) /\left(1+z^{2}\right) \sqrt{1+z^{2}-p^{2} .}
\end{aligned}
$$

All the equations of the family generate new conserved quantities. For example, equations (13a), (13b) can be written as

$$
\left(\psi_{x}+\psi_{t}^{2}\right)_{x}=\left(\psi_{x} \psi_{t}\right)_{t} ; \quad\left(\chi_{t}-\chi_{x}^{2}\right)_{t}=\left(-\chi_{t} \chi_{x}\right)_{x}
$$

and by means of (10a), (10b) new currents can be obtained for the original Born - Infeld equation (1):

$$
\begin{aligned}
& F^{6}(z, p)=2 z p\left(1+z^{2}\right) /\left(1-p^{2}\right)^{2} \\
& G^{6}(z, p)=\left[\left(1+z^{2}\right)\left(1-p^{2}\right)+4 z^{2} p^{2}\right] /\left(1-p^{2}\right)^{2} \\
& F^{6}(z, p)=\left[\left(p^{2}-1\right)\left(1+z^{2}\right)-4 z^{2} p^{2}\right] /\left(1+z^{2}\right)^{2} \\
& G^{6}(z, p)=-2 z p\left(p^{2}-1\right) /\left(1+z^{2}\right)^{2} .
\end{aligned}
$$


At last let us introduce an infinite set of currents for (1). For equations (15a) and (15b) the quantities $(F, G)$ and $(\bar{F}, \bar{G})$ must satisfy, respectively, the equations

$$
G_{b}-F_{a}=2 b F_{b} / a, \quad G_{a}=\left(1-b^{2}\right) F_{b} / a^{2}
$$

and

$$
\bar{G}_{d}-\bar{F}_{c}=-2 c \bar{G}_{c} / d, \quad \bar{F}_{d}=\left(1-c^{2}\right) \bar{G}_{c} / d^{2},
$$

where $a=\mu_{x}, b=\mu_{t}, c=v_{x}, d=v_{t}$.

Eliminating $F$ and $\bar{G}$ we get

$$
\begin{aligned}
& a^{2} F_{a a}+2 a b F_{a b}+\left(b^{2}-1\right) F_{b b}=0, \\
& d^{2} \bar{G}_{d d}+2 c d \bar{G}_{c d}+\left(c^{2}-1\right) \bar{G}_{c c}=0 .
\end{aligned}
$$

Introducing new variables

$$
\xi=b / a=z p /\left(1+z^{2}\right), \quad \eta=1 / a=\sqrt{1+z^{2}-p^{2}} /\left(1+z^{2}\right)
$$

and

$$
\bar{\xi}=c / d=z p /\left(p^{2}-1\right), \quad \bar{\eta}=1 / d=\sqrt{1+z^{2}-p^{2}} /\left(p^{2}-1\right),
$$

we get, respectively,

$$
\begin{aligned}
& F_{\xi \xi}-F_{\eta \eta}=2 F_{\eta} / \eta, \\
& \bar{G}_{\bar{\xi} \bar{\xi}}-\bar{G}_{\bar{\eta}} \bar{\eta}_{\eta}=2 \bar{G}_{\bar{\eta}} / \bar{\eta} .
\end{aligned}
$$

The general solution of these equations is

$$
R(\xi, \eta)=[\varphi(\xi+\eta)+\psi(\xi-\eta)] / \eta,
$$

where $\varphi$ and $\psi$ are the arbitrary functions.

So, finding $G$ and $\bar{F}$ we can write

$$
\begin{aligned}
& F=[\varphi(\xi+\eta)+\psi(\xi-\eta)] / \eta, \\
& G=[(\xi-\eta) \varphi(\xi+\eta)+(\xi+\eta) \psi(\xi-\eta)] / \eta ; \\
& \bar{F}=[(\bar{\xi}-\bar{\eta}) \bar{\varphi}(\bar{\xi}+\bar{\eta})+(\bar{\xi}+\bar{\eta}) \bar{\psi}(\bar{\xi}-\bar{\eta})] / \bar{\eta}, \\
& \bar{G}=[\bar{\varphi}(\bar{\xi}+\bar{\eta})+\bar{\psi}(\bar{\xi}-\bar{\eta})] / \bar{\eta},
\end{aligned}
$$

where $\varphi, \psi, \bar{\varphi}, \bar{\psi}$ are the arbitrary functions.

Now consider $F$ in the form

$$
F=f(\xi) g(\eta)
$$

(similarly $\bar{G}=h(\bar{\xi}) j(\bar{\eta}))$.

Then $f$ and $g$ ( $h$ and $j$ ) are the eigenfunctions of the operators

$$
\begin{array}{cl}
\partial^{2} / \partial \xi^{2}, & \eta\left(\partial^{2} / \partial \eta^{2}\right)+2(\partial / \partial \eta) \\
\left(\partial^{2} / \partial \bar{\xi}^{2},\right. & \left.\bar{\eta}\left(\partial^{2} / \partial \bar{\eta}^{2}\right)+2(\partial / \partial \bar{\eta})\right) .
\end{array}
$$

Hence $f$ and $g$ must be taken in a form

$$
\begin{aligned}
& f(\lambda, \xi)=A(\lambda) \mathrm{e}^{\lambda \xi}+B(\lambda) \mathrm{e}^{-\lambda \xi}, \\
& g(\lambda, \eta)=\left[C(\lambda) \mathrm{e}^{\lambda \eta}+D(\lambda) \mathrm{e}^{-\lambda \eta}\right] / \eta .
\end{aligned}
$$


Then we have

$$
\begin{aligned}
& F=f(\xi) g(\eta) \\
& G=\xi f(\xi) g(\eta)-\eta \bar{f}(\xi) \bar{g}(\eta)
\end{aligned}
$$

(analogous expressions we have for $(\bar{G}, \bar{F}))$, where

$$
\begin{array}{cc}
f(\lambda, \xi)=A(\lambda) \mathrm{e}^{\lambda \xi}+B(\lambda) \mathrm{e}^{-\lambda \xi ;} & \bar{f}(\lambda, \xi)=-A(\lambda) \mathrm{e}^{\lambda \xi}+B(\lambda) \mathrm{e}^{-\lambda \xi} ; \\
g(\lambda, \eta)=\left[C(\lambda) \mathrm{e}^{\lambda \eta}+D(\lambda) \mathrm{e}^{-\lambda \eta}\right] / \eta ; & \bar{g}(\lambda, \eta)=\left[-C(\lambda) \mathrm{e}^{\lambda \eta}+D(\lambda) \mathrm{e}^{-\lambda \eta}\right] / \eta
\end{array}
$$

and $A(\lambda), B(\lambda), C(\lambda), D(\lambda)$ are the arbitrary functions of the eigenvalue $\lambda$.

We are grateful to A. Ainsaar and I. Piir for fruitful discussions.

\title{
REFERENCES
}

1. 3 а х а ро в В. Е., Ф а д де е в Л. Д., Функц. анализ и его прилож., 5, № 4, 18-27 (1971).

2. Барб ашов Б. Н., Черников Н. А., Ж. эксперим. и теорет. физ., 50, вып. 5, $1296-1308$ (1966).

3. E is e nhart, L. P., A treatise on the differential geometry of curves and surfaces, New York, Dover, 1960.

Academy of Sciences of the Estonian SSR, Institute of Physics

Received January 31,1979

\author{
M. KOIV, V. ROSENHAUS
}

\section{KAHEMŌOTMELISTE BORN-INFELDI VORRANDITE PERE JA JÄAVUSSEADUSTE SÜTEEM}

Artikkel käsitleb kahemõõtmelise relativistlikult invariantse Born-Infeldi võrrandi jäävaid suurusi, nn. voole. Viimased jagunevad paarideks, paaride hulgas leidub klass (baasvoolud), mille kaudu genereeritakse Born-Infeldi vorrrandite pere. Baasvoolud määravad teisendused pere lahendite vahel. Peresse kuuluvate võrrandite hulgas on täielikult ja osaliselt hodograafinvariantseid, samuti vōrrandeid, mis lineariseeruvad hodograafteisenduse korral.

\author{
М. КЫИВ, В. РОЗЕНГАУЗ
}

\section{СЕМЕИСТВО ДВУХМЕРНЫХ УРАВНЕНИИ БОРНА-ИНФЕЛЬДА И СИСТЕМА ЗАКОНОВ СОХРАНЕНИЯ}

Рассматриваются сохраняющиеся всличнны низшего порядка - т. н. токи - двухмерного лоренц-инвариантного уравнения Борна-Инфельда. Токи распадаются на пары, которые содержат определенный класс (базисные токи), с помощью которого и находится семейство уравнений Борна-Инфельда. Базисные токи определяют преобразования между решениями его уравнений. Уравнения из данного семейства полностью и частично годограф-инвариантны, а также эффективно линейны (т. е, такие нелинейные уравнения, годограф-преобразованные уравнения которых линейны). 